\title{
The $*$-holonomy group of the Stefan suspension of a diffeomorphism
}

\author{
by ANDRZEJ PiA̧TKOWski (Eódź)
}

\begin{abstract}
The definition of a Stefan suspension of a diffeomorphism is given. If $\mathcal{G}_{g}$ is the Stefan suspension of the diffeomorphism $g$ over a Stefan foliation $\mathcal{G}$, and $G_{0} \in \mathcal{G}$ satisfies the condition $g \mid G_{0}=\operatorname{id}_{G_{0}}$, then we compute the $*$-holonomy group for the leaf $F_{0} \in \mathcal{G}_{g}$ determined by $G_{0}$. A representative element of the $*$-holonomy along the standard imbedding of $S^{1}$ into $F_{0}$ is characterized. A corollary for the case when $G_{0}$ contains only one point is derived.
\end{abstract}

0. Introduction. Our base is the notion of a Stefan foliation introduced in [4]. In the present paper, "*-holonomy" has the same meaning as holonomy defined in [2]. This new terminology is introduced in order to distinguish it from Ehresmann holonomy ([1], [5]).

Let $N$ be a smooth manifold and let $\mathcal{G}$ be a Stefan foliation of $N$. Let $g: N \rightarrow N$ be a diffeomorphism which maps leaves into leaves. In Section 1 we define the Stefan suspension of $g$ over $\mathcal{G}$.

Let $G_{0} \in \mathcal{G}$ satisfy the condition $g \mid G_{0}=\operatorname{id}_{G_{0}}$, let $\mathcal{F}$ be the Stefan suspension of $g$ over $\mathcal{G}$ and let $F_{0} \in \mathcal{F}$ be determined by $G_{0}$. Section 2 contains theorems on the $*$-holonomy group of $F_{0}$. Theorem (2.1) asserts that this group is isomorphic to the product of the $*$-holonomy group of $G_{0}$ and the group generated by the $*$-holonomy along the standard imbedding of $S^{1}$ into $F_{0}$. Theorem (2.2) says that, for an arbitrary transversal $\Sigma$ containing $y_{0} \in G_{0}$, there exists a representative element of the $*$-holonomy conjugate to $g \mid \Sigma$. As a corollary we obtain the following fact: if $G_{0}$ contains the single point $y_{0}$, then the $*$-holonomy group of $F_{0}$ is isomorphic to the group generated by the class of the diffeomorphism $g$.

We adopt the terminology and notation from [2]. The only exception is the symbol $*-\operatorname{Hol}_{x_{0}}(\mathcal{F}, \varphi)$ instead of $\operatorname{Hol}_{x_{0}}(\mathcal{F}, \varphi)$ used in [2].

1991 Mathematics Subject Classification: Primary 57R30.

Key words and phrases: Stefan foliation, suspension, holonomy group. 
1. A Stefan suspension of a diffeomorphism. Let $N$ be a smooth manifold of dimension $n$ and $\mathcal{G}$ a Stefan foliation of $N$. Let $g: N \rightarrow N$ be a diffeomorphism which maps leaves of $\mathcal{G}$ into leaves of $\mathcal{G}$.

In $N \times \mathbb{R}$, define the equivalence relation $\sim$ in the following way: $(y, t) \sim$ $\left(y^{\prime}, t^{\prime}\right)$ if and only if $t-t^{\prime}=k \in \mathbb{Z}$ and $y^{\prime}=g^{k}(y)$. In other words, consider on $N \times \mathbb{R}$ the diffeomorphism $\bar{g}(y, t)=\left(g^{-1}(y), t+1\right)$. Then $(y, t) \sim\left(y^{\prime}, t^{\prime}\right)$ if and only if $\left(y^{\prime}, t^{\prime}\right)=\bar{g}^{k}(y, t)$ for some $k \in \mathbb{Z}$. It is well known that $M:=N \times \mathbb{R} / \sim$ is a manifold of dimension $n+1$ and the canonical projection $\pi: N \times \mathbb{R} \rightarrow M$ is a covering.

Consider in $N \times \mathbb{R}$ a foliation $\mathcal{F}_{0}:=\mathcal{G} \times \widetilde{\mathbb{R}}([5])$ where $\widetilde{\mathbb{R}}$ is the foliation of $\mathbb{R}$ consisting of a single leaf. Note that $\mathcal{F}_{0}$ is invariant under the diffeomorphism $\bar{g}$. It is easy to see that there exists a Stefan foliation $\mathcal{F}$ of $M$ such that $\mathcal{F}_{0}=\pi^{*}(\mathcal{F})([3],[5])$. Leaves of $\mathcal{F}$ are submanifolds of $M$ of the form $\pi(G \times \mathbb{R})$ where $G \in \mathcal{G}$ and the foliation $\mathcal{F}$ is locally isomorphic to $\mathcal{F}_{0}$. The foliation $\mathcal{F}$ is called the Stefan suspension of $g$ over $\mathcal{G}$.

A simple computation proves that the following facts hold:

(1.1) If $\psi$ is a distinguished chart of $\mathcal{G}$ around $y_{0}$, then $\psi \circ g$ is a distinguished chart of this foliation around $g^{-1}\left(y_{0}\right)$ with the domain $g^{-1}\left(D_{\psi}\right)$.

(1.2) If $\psi$ is a distinguished chart of $\mathcal{G}$ around $y_{0}$, and $t_{0} \in \mathbb{R}$, then the mapping

$$
\begin{aligned}
\varphi: \pi\left(D_{\psi} \times\left(t_{0}-1 / 2, t_{0}+1 / 2\right)\right) & \ni \pi(y, t) \\
& \mapsto\left(t-t_{0}, \psi(y)\right) \in(-1 / 2,1 / 2) \times U_{\psi} \times W_{\psi}
\end{aligned}
$$

$\left(y \in D_{\psi}, t \in\left(t_{0}-1 / 2, t_{0}+1 / 2\right)\right)$ is a distinguished chart of $\mathcal{F}$ around $\pi\left(y_{0}, t_{0}\right) \in M$.

Introduce the following notation for the natural projections: $\operatorname{pr}_{1}: U_{\psi} \times$ $W_{\psi} \rightarrow U_{\psi}, \mathrm{pr}_{2}: U_{\psi} \times W_{\psi} \rightarrow W_{\psi}, \operatorname{Pr}_{1}:(-1 / 2,1 / 2) \times U_{\psi} \times W_{\psi} \rightarrow$ $(-1 / 2,1 / 2) \times U_{\psi}=U_{\varphi}$ and $\operatorname{Pr}_{2}:(-1 / 2,1 / 2) \times U_{\psi} \times W_{\psi} \rightarrow W_{\psi}=W_{\varphi}$.

2. The *-holonomy group of a Stefan suspension. Let $G_{0} \in \mathcal{G}$ be a leaf for which

$$
g \mid G_{0}=\operatorname{id}_{G_{0}} .
$$

Let $F_{0}=\pi\left(G_{0} \times \mathbb{R}\right) \in \mathcal{F}$. Note that $F_{0}=G_{0} \times S^{1}$ by (1). Denote by $p_{G_{0}}$ and $p_{S^{1}}$ the natural projections of $F_{0}$ onto $G_{0}$ and $S^{1}$, respectively. We have

$$
\pi_{1}\left(F_{0}\right) \cong \pi_{1}\left(G_{0}\right) \times \pi_{1}\left(S^{1}\right) .
$$

It is easy to check that each element of $\pi_{1}\left(G_{0}\right)$ commutes with each element of $\pi_{1}\left(S^{1}\right)$ in $\pi_{1}\left(F_{0}\right)$.

Let $y_{0} \in G_{0}$ and $x_{0}=\pi\left(y_{0}, 0\right)$. Fix a distinguished chart $\psi$ of $\mathcal{G}$ around $y_{0}$ and let $\varphi$ be the distinguished chart of $\mathcal{F}$ defined as in (1.2) with $t_{0}=0$. 
At the point $x_{0}$ consider the loop

$$
\gamma:[0,1] \ni s \mapsto \pi\left(y_{0}, s\right) \in F_{0} .
$$

Under the above assumptions, we prove the following

(2.1) TheOREM. $*-\mathrm{Hol}_{x_{0}}(\mathcal{F}, \varphi) \cong *-\operatorname{Hol}_{y_{0}}(\mathcal{G}, \psi) \times\left\langle\left[f_{\gamma ; \varphi, \varphi}\right]\right\rangle$.

(Here, $\left\langle\left[f_{\gamma ; \varphi, \varphi}\right]\right\rangle$ denotes the subgroup of $\mathcal{A}_{\varphi} / \equiv$ generated by $\left[f_{\gamma ; \varphi, \varphi}\right]$.)

Pr o of. Define

$$
\Phi: *-\mathrm{Hol}_{y_{0}}(\mathcal{G}, \psi) \times\left\langle\left[f_{\gamma ; \varphi, \varphi}\right]\right\rangle \rightarrow *-\mathrm{Hol}_{x_{0}}(\mathcal{F}, \varphi)
$$

by the formula

$$
\Phi\left(h_{\mathcal{G}, \psi}([\alpha]),\left[f_{\gamma ; \varphi, \varphi}\right]^{k}\right)=h_{\mathcal{F}, \varphi}\left([\bar{\alpha}] \cdot[\gamma]^{k}\right)
$$

(with $h$ being the holonomy homomorphism of the respective foliation), where $k \in \mathbb{Z}, \alpha$ is a loop in $G_{0}$ at $y_{0}$ and $\bar{\alpha}:[0,1] \ni s \mapsto \pi(\alpha(s), 0) \in F_{0}$.

By using chains of charts described in (1.2), it is easy to check that the definition of $\Phi$ is correct. Note that $\Phi$ takes its values in $*-\operatorname{Hol}_{x_{0}}(\mathcal{F}, \varphi)$ by (3).

We show that $\Phi$ is a group homomorphism. Using the commutativity mentioned after (2), we have

$$
\begin{aligned}
h_{\mathcal{F}, \varphi}([\bar{\alpha}]) \cdot\left[f_{\gamma ; \varphi, \varphi}\right]^{k} & =h_{\mathcal{F}, \varphi}([\bar{\alpha}]) \cdot h_{\mathcal{F}, \varphi}\left([\gamma]^{k}\right)=h_{\mathcal{F}, \varphi}\left([\bar{\alpha}] \cdot[\gamma]^{k}\right) \\
& =h_{\mathcal{F}, \varphi}\left([\gamma]^{k} \cdot[\bar{\alpha}]\right)=\left[f_{\gamma ; \varphi, \varphi}\right]^{k} \cdot h_{\mathcal{F}, \varphi}([\bar{\alpha}]) .
\end{aligned}
$$

Therefore, by simple computations, we get

$$
\begin{aligned}
\Phi\left(\left(h_{\mathcal{G}, \psi}([\alpha]),\left[f_{\gamma ; \varphi, \varphi}\right]^{k}\right) \cdot\left(h_{\mathcal{G}, \psi}\left(\left[\alpha^{\prime}\right]\right),\left[f_{\gamma ; \varphi, \varphi}\right]^{k^{\prime}}\right)\right) \\
=\Phi\left(h_{\mathcal{G}, \psi}([\alpha]),\left[f_{\gamma ; \varphi, \varphi}\right]^{k}\right) \cdot \Phi\left(h_{\mathcal{G}, \psi}\left(\left[\alpha^{\prime}\right]\right),\left[f_{\gamma ; \varphi, \varphi}\right]^{k^{\prime}}\right) .
\end{aligned}
$$

Define

$$
\Psi: *-\mathrm{Hol}_{x_{0}}(\mathcal{F}, \varphi) \rightarrow *-\mathrm{Hol}_{y_{0}}(\mathcal{G}, \psi) \times\left\langle\left[f_{\gamma ; \varphi, \varphi}\right]\right\rangle
$$

by the formula

$$
\Psi\left(h_{\mathcal{F}, \varphi}([\delta])\right)=\left(h_{\mathcal{G}, \psi}\left(\left[p_{G_{0}} \circ \delta\right]\right),\left[f_{\gamma ; \varphi, \varphi}\right]^{k}\right)
$$

where $\delta$ is a loop in $F_{0}$ at $x_{0}$ and $k$ is an integer such that $\left[p_{S^{1}} \circ \delta\right]=[\beta]^{k}$ with $\beta:[0,1] \ni s \mapsto e^{2 \pi i s} \in S^{1}$.

We show that the above definition is correct. Fix $\delta$ for a moment. For each $s \in[0,1]$, take an arbitrary distinguished chart $\psi^{(s)}\left(\psi^{(0)}=\psi^{(1)}=\psi\right)$ of $\mathcal{G}$ around $y(s):=p_{G_{0}} \circ \delta(s)$. Let $t:[0,1] \rightarrow \mathbb{R}$ be the unique lift of $p_{S^{1}} \circ \delta$ to the universal covering of $S^{1}$ with $t(0)=0$. Note that

$$
\delta(s)=\pi(y(s), t(s)) .
$$

Define a distinguished chart $\varphi^{(s)}$ around $\delta(s)$ as in (1.2), using the chart $\psi^{(s)}$ and setting $t_{0}=t(s)$. From the family $\left\{\varphi^{(s)}: s \in[0,1]\right\}$ choose a finite 
subfamily $\left\{\varphi_{0}, \varphi_{1}, \ldots, \varphi_{r}\right\}$ (with $\varphi_{0}=\varphi^{(0)}, \varphi_{r}=\varphi^{(1)}$ and $\varphi_{i}=\varphi^{\left(s_{i}\right)}$ for $i=1, \ldots, r-1)$ such that the sequence

$$
\widetilde{\mathcal{C}}=\left(\varphi_{0}, 0 ; \varphi_{1}, s_{1} ; \ldots ; \varphi_{r}, 1 ; \varphi_{0}, 1\right)
$$

is a chain along $\delta$. We prove that the sequence

$$
\mathcal{C}=\left(\psi_{0}, 0 ; \psi_{1}, s_{1} ; \ldots ; \psi_{r}, 1\right)
$$

is a chain along $p_{G_{0}} \circ \delta$, where $\psi_{0}=\psi^{(0)}=\psi^{(1)}=\psi_{r}=\psi$ and $\psi_{i}=\psi^{\left(s_{i}\right)}$ for $i=1, \ldots, r-1$. To this end, we prove

Lemma A. If $\widetilde{s} \in \delta^{-1}\left(D_{\varphi_{i}}\right)_{s_{i}}$ (the connected component of $\delta^{-1}\left(D_{\varphi_{i}}\right)$ containing $\left.s_{i}\right)$, then $t(\widetilde{s}) \in\left(t\left(s_{i}\right)-1 / 2, t\left(s_{i}\right)+1 / 2\right)$.

P r o of. It follows directly from the definitions of $\varphi_{i}, t$ and from (5) that

$$
t\left(\delta^{-1}\left(D_{\varphi_{i}}\right)_{s_{i}}\right) \subset\left(t\left(s_{i}\right)-1 / 2, t\left(s_{i}\right)+1 / 2\right) .
$$

In particular, $t(\widetilde{s}) \in\left(t\left(s_{i}\right)-1 / 2, t\left(s_{i}\right)+1 / 2\right)$.

Lemma A implies

Lemma B. $\delta^{-1}\left(D_{\varphi_{i}}\right)_{s_{i}} \subset\left(p_{G_{0}} \circ \delta\right)^{-1}\left(D_{\psi_{i}}\right)_{s_{i}}$.

Proof. If $\widetilde{s} \in \delta^{-1}\left(D_{\varphi_{i}}\right)_{s_{i}}$, then $\pi(y(\widetilde{s}), t(\widetilde{s}))=\pi\left(y^{\prime}, t^{\prime}\right)$ for some $y^{\prime} \in$ $D_{\psi_{i}}, t^{\prime} \in\left(t\left(s_{i}\right)-1 / 2, t\left(s_{i}\right)+1 / 2\right)$ and, using Lemma A, we obtain $y(\widetilde{s})=$ $\left(p_{G_{0}} \circ \delta\right)(\widetilde{s}) \in D_{\psi_{i}}$, which gives the assertion.

Directly from Lemma B it follows that if $\delta^{-1}\left(D_{\varphi_{i}}\right)_{s_{i}} \cap \delta^{-1}\left(D_{\varphi_{i+1}}\right)_{s_{i+1}} \neq$ $\emptyset$, then $\left(p_{G_{0}} \circ \delta\right)^{-1}\left(D_{\psi_{i}}\right)_{s_{i}} \cap\left(p_{G_{0}} \circ \delta\right)^{-1}\left(D_{\psi_{i+1}}\right)_{s_{i+1}} \neq \emptyset$. Thus $\mathcal{C}$ is a chain along $p_{G_{0}} \circ \delta$.

We now show that the $*$-holonomy diffeomorphism determined by the part

$$
\left(\varphi_{0}, 0 ; \varphi_{1}, s_{1} ; \ldots ; \varphi_{r}, 1\right)
$$

of $\widetilde{\mathcal{C}}$ is equal to $f_{\mathcal{C}}$. Indeed, let $\widetilde{s}_{i} \in \delta^{-1}\left(D_{\varphi_{i}}\right)_{s_{i}} \cap \delta^{-1}\left(D_{\varphi_{i+1}}\right)_{s_{i+1}}, i=$ $0,1, \ldots, r-1$. Then, by Lemmas $\mathrm{A}$ and $\mathrm{B}$, we have

$$
f_{\varphi_{i}, \varphi_{i+1} ; \delta\left(\tilde{s}_{i}\right)}(w)=f_{\psi_{i}, \psi_{i+1} ; y\left(\tilde{s}_{i}\right)}(w) .
$$

Suppose now that

$$
h_{\mathcal{F}, \varphi}([\delta])=h_{\mathcal{F}, \varphi}\left(\left[\delta^{\prime}\right]\right) .
$$

Take chains $\widetilde{\mathcal{C}}$ and $\widetilde{\mathcal{C}}^{\prime}$ constructed as above along $\delta$ and $\delta^{\prime}$, respectively. Then $f_{\widetilde{\mathcal{C}}} \equiv f_{\widetilde{\mathcal{C}}^{\prime}}$. Along the curve $\eta=\delta * \delta^{\prime-1}$ we can construct a chain $\overline{\mathcal{C}}$ by composing links of $\widetilde{\mathcal{C}}$ and links of $\widetilde{\mathcal{C}^{\prime}}$ in opposite order. We have

$$
\begin{aligned}
\overline{\mathcal{C}}= & \left(\varphi_{0}, 0 ; \varphi_{1},(1 / 2) s_{1} ; \ldots ; \varphi_{r}, 1 / 2 ; \varphi_{0}, 1 / 2 ; \varphi_{0}, 1 / 2 ;\right. \\
& \left.\varphi_{r^{\prime}}^{\prime}, 1 / 2 ; \varphi_{r^{\prime}-1}^{\prime}, 1-(1 / 2) s_{r^{\prime}-1}^{\prime} ; \ldots ; \varphi_{1}^{\prime}, 1-(1 / 2) s_{1}^{\prime} ; \varphi_{0}, 1\right) .
\end{aligned}
$$


By [2], the *-holonomy does not depend on the choice of the chain. We can cross out in $\overline{\mathcal{C}}$ two links of the form $\left(\varphi_{0}, 1 / 2\right)$. We get the chain

$$
\overline{\overline{\mathcal{C}}}=\left(\varphi_{0}, 0 ; \varphi_{1},(1 / 2) s_{1} ; \ldots ; \varphi_{r}, 1 / 2 ; \varphi_{r^{\prime}}^{\prime}, 1 / 2 ; \ldots ; \varphi_{1}^{\prime}, 1-(1 / 2) s_{1}^{\prime} ; \varphi_{0}, 1\right) .
$$

Then

$$
f_{\mathcal{C}^{\prime}}^{-1} \circ f_{\mathcal{C}}=f_{\overline{\overline{\mathcal{C}}}} \equiv f_{\overline{\mathcal{C}}}=f_{\widetilde{\mathcal{C}}^{\prime}}^{-1} \circ f_{\widetilde{\mathcal{C}}} \equiv \operatorname{id}_{W}
$$

by $(6)$. Thus $f_{\mathcal{C}} \equiv f_{\mathcal{C}^{\prime}}$, so

$$
h_{\mathcal{G}, \psi}\left(\left[p_{G_{0}} \circ \delta\right]\right)=h_{\mathcal{G}, \psi}\left(\left[p_{G_{0}} \circ \delta^{\prime}\right]\right) .
$$

Therefore, the first coordinate of $\Psi$ is correctly defined.

Note that from the properties of the isomorphism $\zeta: \pi_{1}\left(F_{0}, x_{0}\right) \rightarrow$ $\pi_{1}\left(G_{0}, y_{0}\right) \times \pi_{1}\left(S^{1}, 1\right)$ it follows that

$$
[\delta]=\left[\overline{p_{G_{0}} \circ \delta}\right] \cdot\left[\overline{p_{S^{1}} \circ \delta}\right]
$$

for every loop $\delta$ in $F_{0}$ at $x_{0}$, where, for arbitrary curves $\alpha:[0,1] \rightarrow G_{0}, \varepsilon$ : $[0,1] \rightarrow S^{1}$, we define $\bar{\alpha}:[0,1] \ni s \mapsto \pi(\alpha(s), 0) \in F_{0}$ and $\bar{\varepsilon}:[0,1] \ni s \mapsto$ $\left(y_{0}, \varepsilon(s)\right) \in G_{0} \times S^{1}=F_{0}$.

Since $h_{\mathcal{F}, \varphi}$ is a homomorphism, (7) implies

(10) $h_{\mathcal{F}, \varphi}\left(\left[\overline{p_{G_{0}} \circ \delta}\right]\right) \cdot h_{\mathcal{F}, \varphi}\left(\left[\overline{p_{S^{1}} \circ \delta}\right]\right)=h_{\mathcal{F}, \varphi}\left(\left[\overline{p_{G_{0}} \circ \delta^{\prime}}\right]\right) \cdot h_{\mathcal{F}, \varphi}\left(\left[\overline{p_{S^{1}} \circ \delta^{\prime}}\right]\right)$.

We have

$$
h_{\mathcal{G}, \psi}\left(\left[p_{G_{0}} \circ \delta\right]\right)=h_{\mathcal{G}, \psi}\left(\left[p_{G_{0}} \circ \delta^{\prime}\right]\right)
$$

by (8). It follows that $h_{\mathcal{F}, \varphi}\left(\left[\overline{p_{G_{0}} \circ \delta}\right]\right)=h_{\mathcal{F}, \varphi}\left(\left[\overline{p_{G_{0}} \circ \delta^{\prime}}\right]\right)$. Thus, multiplying (10) by the inverse of $h_{\mathcal{F}, \varphi}\left(\left[\overline{p_{G_{0}} \circ \delta}\right]\right)$, we obtain

$$
h_{\mathcal{F}, \varphi}\left(\left[\overline{p_{S^{1}} \circ \delta}\right]\right)=h_{\mathcal{F}, \varphi}\left(\left[\overline{p_{S^{1}} \circ \delta^{\prime}}\right]\right)
$$

which means that

$$
\left[f_{\gamma ; \varphi, \varphi}\right]^{k}=\left[f_{\gamma ; \varphi, \varphi}\right]^{k^{\prime}}
$$

where $k, k^{\prime}$ are integers such that $\left[p_{S^{1}} \circ \delta\right]=[\beta]^{k},\left[p_{S^{1}} \circ \delta^{\prime}\right]=[\beta]^{k^{\prime}}$. Consequently, the second coordinate of $\Psi$ is correctly defined.

It is easy to check that $\Psi$ is the inverse of $\Phi$.

Let $\Sigma$ be an arbitrary transversal of $\mathcal{G}$ containing $y_{0}([5])$. Then $\Sigma^{\prime}=$ $g(\Sigma)$ is a transversal of $\mathcal{G}$ containing $y_{0}$. We have

(2.2) THEOREM. There exist a distinguished chart $\varphi$ of $\mathcal{F}$ around $x_{0}$ and a chain $\widetilde{\mathcal{C}} \in C_{\varphi, \varphi}^{\gamma}$ such that the diagram

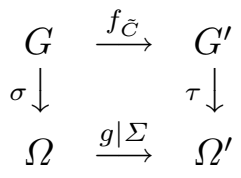


commutes. Here $G, G^{\prime}$ are open neighbourhoods of 0 in $W_{\varphi}, \Omega, \Omega^{\prime}$ are open neighbourhoods of $y_{0}$ in $\Sigma, \Sigma^{\prime}$, respectively, and the vertical mappings are diffeomorphisms compatible with the induced foliations.

Pr o of. Let $x_{0}=\pi\left(y_{0}, 0\right)$. Take a distinguished chart $\psi$ of $\mathcal{G}$ around $y_{0}$ such that $\psi^{-1}\left(\{0\} \times W_{\psi}\right) \subset \Sigma([2])$. Then $\psi^{\prime}=\psi \circ g$ is a distinguished chart of $\mathcal{G}$ around $y_{0}$ by (1.1). Set

$$
\widetilde{\mathcal{C}}=\left(\varphi, 0 ; \varphi^{\prime}, 1 / 2 ; \varphi, 1\right)
$$

where $\varphi$ and $\varphi^{\prime}$ are defined by

$$
\begin{gathered}
\varphi: \pi\left(D_{\psi} \times(-1 / 2,1 / 2)\right) \ni \pi(y, t) \mapsto(t, \psi(y)) \in(-1 / 2,1 / 2) \times U_{\psi} \times W_{\psi}, \\
\varphi^{\prime}: \pi\left(D_{\psi^{\prime}} \times(0,1)\right) \ni \pi(y, t) \mapsto\left(t-1 / 2, \psi^{\prime}(y)\right) \in(-1 / 2,1 / 2) \times U_{\psi} \times W_{\psi} .
\end{gathered}
$$

We show that $\widetilde{\mathcal{C}}$ is a chain along $\gamma$. Obviously, $\varphi$ is a chart around $\gamma(0)=\gamma(1)$ and $\varphi^{\prime}$ is a chart around $\gamma(1 / 2)$. Thus all three terms of $\widetilde{\mathcal{C}}$ are links. Since

$$
\gamma^{-1}\left(D_{\varphi}\right)=[0,1 / 2) \cup(1 / 2,1] \text { and } \gamma^{-1}\left(D_{\varphi^{\prime}}\right)=(0,1),
$$

we have

$$
\begin{aligned}
& \gamma^{-1}\left(D_{\varphi}\right)_{0} \cap \gamma^{-1}\left(D_{\varphi^{\prime}}\right)_{1 / 2}=(0,1 / 2) \neq \emptyset, \\
& \gamma^{-1}\left(D_{\varphi^{\prime}}\right)_{1 / 2} \cap \gamma^{-1}\left(D_{\varphi}\right)_{1}=(1 / 2,1) \neq \emptyset .
\end{aligned}
$$

In order to define a $*$-holonomy diffeomorphism, take the points $\gamma(1 / 4)$ and $\gamma(3 / 4)$. By the definition of $\varphi$ and $\varphi^{\prime}$ we have

$$
\begin{aligned}
f_{\widetilde{\mathcal{C}}}(w) & =\operatorname{Pr}_{2} \varphi \varphi^{\prime-1}\left(\operatorname{Pr}_{1} \varphi^{\prime} \gamma(3 / 4), \operatorname{Pr}_{2} \varphi^{\prime} \varphi^{-1}\left(\operatorname{Pr}_{1} \varphi \gamma(1 / 4), w\right)\right) \\
& =\operatorname{pr}_{2} \psi g \psi^{-1}(0, w) .
\end{aligned}
$$

It is easy to check that the mappings $\sigma: W_{\psi} \ni w \mapsto \psi^{-1}(0, w) \in \Sigma$ and $\operatorname{pr}_{2} \psi \mid \Sigma^{\prime}$ are regular at 0 and $y_{0}$, respectively, by the transversality of $\Sigma$ and $\Sigma^{\prime}$. Consequently, there exist open neighbourhoods $G, G^{\prime}$ of 0 in $W_{\varphi}$ and $\Omega, \Omega^{\prime}$ of $y_{0}$ in $\Sigma$ and $\Sigma^{\prime}$, respectively, such that $\sigma$ is a diffeomorphism of $G$ onto $\Omega$ and $\operatorname{pr}_{2} \psi \mid \Sigma^{\prime}$ is a diffeomorphism of $\Omega^{\prime}$ onto $G^{\prime}$. Set $\tau=\left(\operatorname{pr}_{2} \psi \mid \Omega^{\prime}\right)^{-1}$. The diffeomorphisms $\sigma$ and $\tau$ are compatible with the induced foliations since $\psi$ is a distinguished chart.

By (12), we have the commutativity of diagram (11).

Consider the case when $G_{0}=\left\{y_{0}\right\}$. Let $\mathcal{A}$ be the set of all diffeomorphisms $k: U \rightarrow V\left(U, V\right.$ are open neighbourhoods of $y_{0}$ in $\left.N\right)$ such that $k\left(y_{0}\right)=y_{0}$ and $k$ is compatible with the foliations $\mathcal{G} \mid U$ and $\mathcal{G} \mid V$. In $\mathcal{A}$ we introduce the relation $\equiv$ quite analogously to that in $\mathcal{A}_{\varphi, \varphi}([2])$. Then the set $\mathcal{A} / \equiv$ with multiplication determined by superposition of diffeomorphisms is a group. Moreover, note that $g \in \mathcal{A}$. From Theorems (2.1) and (2.2) we immediately get 
(2.4) Corollary. If $G_{0}=\left\{y_{0}\right\}$ and $g\left(y_{0}\right)=y_{0}$, then $*-\mathrm{Hol}_{x_{0}}(\mathcal{F}, \varphi)$ is isomorphic to the subgroup of $\mathcal{A} / \equiv$ generated by the equivalence class of the diffeomorphism $g$.

\section{References}

[1] C. Ehresmann, Structures feuilletées, in: Proc. 5th Canad. Math. Congress, Montréal 1961, 109-172.

[2] A. Piątkowski, A stability theorem for foliations with singularities, Dissertationes Math. 267 (1988).

[3] - On the *-holonomy of the inverse image of a Stefan foliation, Acta Univ. Lodz. Folia Math., to appear.

[4] P. Stefan, Accessible sets, orbits and foliations with singularities, Proc. London Math. Soc. 29 (1974), 699-713.

[5] P. Ver Eecke, Le groupoïde fondamental d'un feuilletage de Stefan, Publ. Sem. Mat. García de Galdeano, Ser. II, Sec. 3, No. 6, Universidad de Zaragoza, 1986.

INSTITUTE OF MATHEMATICS

ŁÓDŹ UNIVERSITY

BANACHA 22

90-238 ŁÓDŹ, POLAND

E-mail: ANDPIAT@PLUNLO51.BITNET

Reçu par la Rédaction le 15.2.1992

Révisé le 28.8.1992 